\title{
Stefan Gerlach, Handlung bei Schelling
}

\section{Fernando Wirtz}

\section{(2penEdition}

\section{Journals}

\section{Edición electrónica}

URL: https://journals.openedition.org/ref/1748

DOI: $10.4000 /$ ref. 1748

ISSN: 2258-014X

\section{Editor}

EuroPhilosophie Editions

Referencia electrónica

Fernando Wirtz, «Stefan Gerlach, Handlung bei Schelling», Revista de Estud(i)os sobre Fichte [En línea] 21 | 2020, Publicado el 01 diciembre 2020, consultado el 05 marzo 2022. URL: http:// journals.openedition.org/ref/1748 ; DOI: https://doi.org/10.4000/ref.1748

Este documento fue generado automáticamente el 5 marzo 2022.

(c) EuroPhilosophie 


\title{
Stefan Gerlach, Handlung bei Schelling
}

\author{
Fernando Wirtz
}

\section{REFERENCIA}

Gerlach, S., Handlung bei Schelling: Zur Fundamentaltheorie von Praxis, Zeit und Religion im mittleren und späten Werk (Philosophische Abhandlungen 117), Frankfurt am Main:

Klostermann, 2019, 390 pp., ISBN 978-3-465-04393-5

1 Stefan Gerlach, salda en este libro dos importantes ausencias latentes en la Schellingforschung contemporánea. En primer lugar, y principalmente, su libro revindica la dimensión práctica de la filosofía de Schelling, partiendo de una convicción que Schelling mismo no deja de repetir: los principios últimos de la filosofía son esencialmente prácticos. Ahora bien, Gerlach al mismo tiempo y con precisión filológica aplica esta perspectiva práctica sobre la filosofía tardía, un área de la obra de Schelling que permanece relativamente poco estudiada (en parte debido a que se trata de textos publicados póstumamente). De esta forma, este libro ofrece una lectura novedosa de Schelling, tomando a la filosofía positiva por las astas.

2 La tarea de esta investigación consiste en dilucidar precisamente la concepción schellingueana de la acción tal y cómo subyace en la obra de Schelling a partir de 1809. Para esto, Gerlach se sirve de su conocimiento de los autores de la llamada Philosophy of Action de influencia anglosajona. Es en las Investigaciones sobre la libertad del hombre (1809) que es posible encontrar ya un punto de inflexión en la filosofía de Schelling. Pues no sólo se establece allí el acto libre de creación de Dios como principio ontológico central, que luego, a partir de 1827, adquiere el estatus sistemático de lo específicamente 'positivo' de la filosofía positiva. Es también allí donde la dualidad interna de Dios, su interna discontinuidad entre fundamento y existencia, hace posible una dinámica novedosa, según la cual un acto libre en Dios se vuelve posible. "Mientras que en la filosofía temprana predominaba la búsqueda de un principio último que 
correspondiera a los requisitos del sistema de la filosofía, en la filosofía tardía predomina la cuestión de las precondiciones últimas del ser en un impulso ontológico" (31).

3 En primer lugar, el libro investiga la practicidad interna de la conciencia y su orientación transitiva hacia las acciones externas (capítulo 1). Para eso de muestra cómo la doctrina de las potencias presenta la estructura de la voluntad y la consciencia. Aquí, el autor reconstruye la genealogía de esta practicidad, partiendo del Sich-selbstSetzen des Ich de la filosofía temprana de influencia fichteana, hasta llegar a las potencias dinámicas del Dios vivo de la filosofía tardía. En ese sentido, no es difícil ver que Schelling mismo define a las potencias en términos volitivos. La primera potencia es aquella que puede-ser (Seinkönnende) y, por lo tanto, la "voluntad que puede querer" (45). Es por ello que para Schelling la primera potencia desempeña el papel del egoísmo (un rasgo presente claramente ya en las Lecciones de Stuttgart): "La voluntad quiere otra cosa y a sí misma como aquello que quiere otra cosa" (46). Esta fuerza expansiva necesita un límite. Así, la segunda potencia es caracterizada como la voluntad universal. Esta potencia es la contracara de la primera potencia, ella es un querer que no se quiere a sí mismo, y por lo tanto es un querer universal (47). Ambas potencias se reúnen, en un gesto típicamente schellingueano, en la tercera potencia, la del espíritu, que es la potencia relacional, fruto de la reciprocidad entre la primera y segunda potencia, pero a su vez, libre con respecto a ellas (por ello el espíritu en la filosofía tardía es sinónimo de libertad).

4 Una vez explicados los principios teóricos del sistema positivo en abstracto, las siguientes secciones del libro examinan su aplicación a la acción divina (capítulos 2 y 3). La creación funciona en sí como paradigma para pensar una filosofía de la acción: ¿Cuál es el fin y el motivo que mueven la acción divina? Es claro que el Dios omnisciente debe saber lo que hace y cuáles son las consecuencias de su hacer. También es cierto que, para Schelling, Dios bien podría permanecer "en sí mismo", es decir, es libre de no crear, pues Dios es, desde la eternidad, perfecto y autosuficiente. Por ello, el fin de la creación no puede ni complementar a Dios, ni representar un plus cognitivo (pues Dios conoce todo eternamente). Es aquí que Gerlach introduce una reflexión importante a partir de la teoría de la acción: "Este 'motivo real' (SW XIII, 277) debe estar en la creación misma. Dado que no hay nada en la situación de creación fuera de la situación de la creación, el acto de la creación no puede en ningún caso haber sido provocado por motivos heterogéneos, es decir, por motivos que estarían fuera de Dios o de la creación. Debe estar motivado por sí mismo" (84). Así "En la creación, el resultado de la acción coincide con su consumación, motivo y meta" (85). Esto es también así porque Schelling piensa el universo como creatio perpetua y no como un sistema cerrado capaz de funcionar como mero medio para un fin. "Con las acciones se dan necesariamente intenciones cuyo contenido son objetivos [Ziele]. En la creación como motivo y objetivo de la acción intencional de la creación, esto se presenta en su forma pura. Así, la concepción de Schelling sobre la acción de Dios corresponde en esencia a una teoría teleológica de la acción, es decir, una teoría que supone que las acciones son primaria e irreductiblemente intencionadas" (87).

5 En el capítulo 4 se introduce el problema antropológico: ¿Cómo se relaciona esta teoría de la libertad de Dios con los seres humanos? Si bien la afirmación sobre el motivo de la creación no agota la pregunta por la razón última de ésta, la explicación de Gerlach ofrece una perspectiva extremadamente útil para pensar el papel del hombre en la 
filosofía positiva. Schelling no es considerado un autor de filosofía práctica, sin embargo, es posible establecer algunos paralelos con respecto a la acción de la creación y a la acción humana. No sólo el ser humano depende de la acción divina, sino que el destino de la creación depende en buena medida de la acción humana. Este es, por así decirlo, el misterio de la teodicea schellingueana: Dios crea al ser humano y le da la posibilidad de poner en marcha una segunda creación, una creación motorizada únicamente por su propia acción. Así, para pensar la historia humana, Gerlach debe recurrir de a la problemática del tiempo. "El acto mismo de la creación está intrínsecamente entrelazado con el problema del tiempo; sólo en él y desde su horizonte de eternidad nace el tiempo" (129). Es en el marco de una temporalidad teológica que prioriza el futuro que es posible pensar la libertad humana. "El hecho de que la acción humana pueda concebirse, en principio, teleológicamente en el marco de una historia igualmente orientada a fines, incluso escatológica, se debe a este momento futuro ya inherente al primer movimiento divino del ser hacia la realidad" (191).

De todas formas, la función del ser humano permanece incompleta sin la dimensión de su conciencia religiosa, eje de orientación fundamental de su acción histórica (cap. 5). Después de todo, Schelling define al ser humano esencialmente como das Gottsetzende, es decir, como el encargado de 'poner' a Dios. Gerlach retoma un poco después la metáfora schellingueana del actor: si bien las líneas del actor fueron escritas previamente por el poeta (Dios), el actor siempre mantiene la libertad de determinar cómo ha de actuar. A partir de aquí se considera el aspecto de la libertad humana por separado desde la perspectiva de la teoría de la acción (capítulo 6) y luego se presenta la teología de Schelling sobre la acción humana bajo sus aspectos particulares (capítulo 7). Vale aquí una mención especial para un pequeño pero rico excursus sobre la filosofía moral de Schelling, un aspecto de su filosofía que, como ya se dijo, suele quedar relegado por parte de la literatura secundaria (290-303).

7 La cuestión sistémica del significado de la teoría de la acción para la filosofía positiva de Schelling conforma la última parte de la investigación (cap. 8). "Puesto que, según la tesis de la filosofía positiva, el fundamento último del mundo consiste en la acción libre de Dios, ésta, a su vez, debe consistir, como medio para su fin, en una superación de su negatividad interior, que se representa en la dilatación temporal del proceso-mundo" (325). Como se vio, uno de los momentos centrales de la filosofía positiva es la creación del mundo. En efecto, es en la creación donde la "positividad" de la filosofía tardía se manifiesta. En contra de la filosofía negativa, aquella que permanece en el reino de la posibilidad conceptual, la filosofía positiva intenta explicar la existencia del mundo a partir de una acción libre. Dios crea algo 'nuevo', algo que no se encontraba presente en la constelación original de su esencia. El mundo no puede derivarse lógicamente del concepto de Dios, no es una necesidad metafísica de Dios crear el mundo. Este punto resulta ciertamente incómodo para cierto racionalismo teológico. La filosofía negativa es capaz de explicar las potencias de Dios en su inmanencia. Sin embargo, sólo la filosofía positiva 'muestra' cómo la creación se da en tanto acción. Más aún, es sólo por medio de la positividad de la acción humana que la obra de Dios puede ser revelada en su totalidad: Dios y el ser humano se encuentran en desarrollo continuo (332). Este capítulo resulta así extremadamente importante para pensar la actualidad del pensamiento de Schelling, por ejemplo, en lo que refiere al debate entre compatibilistas e incompatibilistas (en este caso en referencia a la pregunta de cómo pueden ser compatibles providencia y libertad en Dios). 
8 El libro de Gerlach ofrece así no sólo una novedosa perspectiva en cuanto a la forma de entender acción, temporalidad y antropología en la filosofía idealista, sino también un excelente manual de consulta sobre la filosofía tardía de Schelling. No es común que los comentaristas asuman el desafío de la filosofía positiva tomando en serio sus premisas. En general, las exposiciones sobre la filosofía positiva recuerdan justamente a la filosofía negativa criticada por Schelling. Es decir, usualmente se explica la estructura de la potencia de la Filosofía de la revelación de un modo formal y lógico. Pero la empresa de Schelling radica precisamente en mostrar los límites de la lógica inmanente, señalando cómo es que la acción libre posibilita en última instancia un pensamiento abierto de la historia. Este libro puede considerarse, por lo tanto, como uno de los estudios sobre la filosofía tardía de Schelling más novedosos y a la vez fieles al pensamiento del filósofo, que se publicaron en los últimos años. 